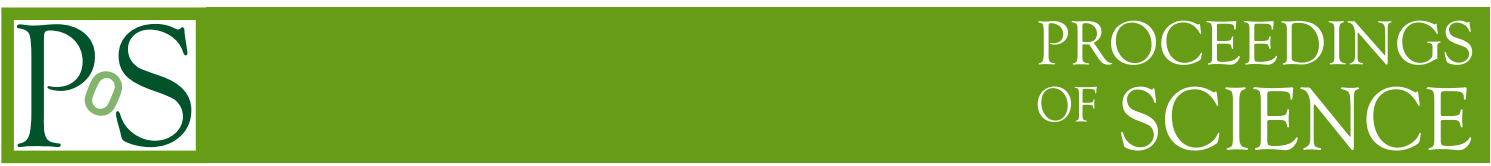

\title{
New 50 cm Photo-Detectors for Hyper-Kamiokande
}

\author{
Yasuhiro NISHIMURA* \\ Institute for Cosmic Ray Research, The University of Tokyo \\ E-mail: vnisidicrr.u-tokyo.ac.ip \\ on behalf of the Hyper-Kamiokande Proto-Collaboration
}

Two types of $50 \mathrm{~cm}$-diameter photo-detectors were developed for Hyper-Kamiokande, a large water Cherenkov detector planned in Japan for neutrino and nucleon decay researches. A boxand-line dynode enabled further improvements on a $50 \mathrm{~cm}$ photomultiplier tube (PMT) used in Super-Kamiokande, which was developed to be an R12860 type by Hamamatsu Photonics K.K. (Hamamatsu). The collection efficiency reached 95\%, superior to 67\% of the PMT in SuperKamiokande. It also gave a higher quantum efficiency of 30\% typical at peak than $22 \%$ in case of the Super-Kamiokande. Another one is a $50 \mathrm{~cm}$-diameter hybrid photo-detector (HPD), R12850 by Hamamatsu, using an avalanche diode for the amplification system. The detection efficiency is as high as the improved PMT, while the resolutions are highest among the existing $50 \mathrm{~cm}$ photo-detectors. At single photoelectron, charge resolutions were evaluated to be $35 \%$ and $15 \%$ in standard deviation and time resolutions to be 4.1 and $3.6 \mathrm{nsec}$ in FWHM for the improved PMT and HPD, respectively. The glass bulb of both new photo-detectors sustained high pressure water up to 1.25 MPa. A newly developed cover for a long-term safe operation in Hyper-Kamiokande was validated at the $80 \mathrm{~m}$ deep water level even with a bulb implosion.

38th International Conference on High Energy Physics

3-10 August 2016

Chicago, USA

* Speaker. 


\section{Introduction}

Hyper-Kamiokande (Hyper-K) is a next generation water Cherenkov detector planned in Japan [四, as an upgrade of Super-Kamiokande (Super-K) [వ]. The total (fiducial) volume is $0.26(0.19)$ million metric tons of water in one of the two tanks in the Hyper-K design. The fiducial volume with two tanks is 17 times larger than that of Super-K. Each tank is optically divided into two layers of inner and outer detectors to reject backgrounds from outside.

A $50 \mathrm{~cm}$-diameter photomultiplier tube (PMT) was initially developed for Kamiokande [ [ $]$, and improved for Super-K [团], where a Venetian blind dynode was adopted to get a wide acceptance. With successful improvements, a $50 \mathrm{~cm}$ PMT with a box-and-line dynode (Box\&Line PMT, R12860 Hamamatsu) was developed. Hyper-K is supposed to use 40,000 Box\&Line PMTs for the inner detector, and 6,700 PMTs with $20 \mathrm{~cm}$ for the outer detectors. A $50 \mathrm{~cm}$ hybrid photo-detector (HPD, R12850 Hamamatsu) equipped with a $20 \mathrm{~mm}$-diameter avalanche diode (AD) was also developed as an alternative option to reach excellent charge and time resolutions among all of existing $50 \mathrm{~cm}$ photo-detectors.

\section{Design of $50 \mathrm{~cm}$ Photo-Detectors}

Table $\mathbb{W}$ lists a design of the two new photo-detectors, compared with that used in Super-K (Super-K PMT, R3600 Hamamatsu). The size such as a diameter and length is almost the same among all photo-detectors, while its curvature was optimized to achieve a high pressure tolerance for the use in the $60 \mathrm{~m}$ water depth of the Hyper-K tank.

Because the electron multiplication path of the Super-K PMT varies in the Venetian blind dynode such that electrons skip one of dynodes, the detection timing and efficiency become worse. The Box\&Line PMT realized a high collection efficiency of photoelectrons (PEs) at the first boxshape dynode, and narrow timing variation by line-focused dynodes.

A typical bias voltage to get $10^{7}$ gain is $2,000 \mathrm{~V}$ for both two types of PMTs, while 8,000 V is required for the HPD. The bombardment gain of the Box\&Line PMT at the first amplification of PEs is about 16, while 1,600 in case of the HPD. This high gain at the first stage is the reason why the HPD brings high resolutions. With an avalanche gain of the AD by a factor of about 50 100 at a $500 \mathrm{~V}$ bias, the total gain of the HPD is $10^{5}$ typically, and reaches to a similar output level as PMTs with a help of preamplifier. The prototype of the HPD is equipped with the preamplifier and high-voltage power supply in a waterproof housing around the pins, so that it can be operated under $10 \mathrm{~V}$ low voltage. A waterproof cable complex with high-voltage lines and connector used up to a $100 \mathrm{~m}$ water depth were also developed as another option for a low heat radiation.

\section{Performance}

\subsection{Detection Efficiency}

The quantum efficiency (QE) of both two new photo-detectors shows $30-36 \%$ at peak wave-

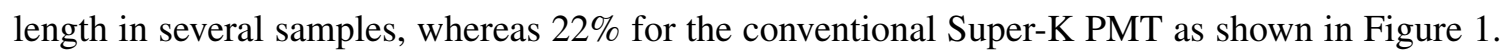
It is noted that recent products of the Box\&Line PMT show similar 35\% level like the HPD's. 
Table 1: Specification of three $50 \mathrm{~cm}$ diameter photo-detectors by Hamamatsu. The values in the bottom line show the collection efficiency (CE), single PE time resolution in FWHM and the typical bias voltage provided from Hamamatsu.

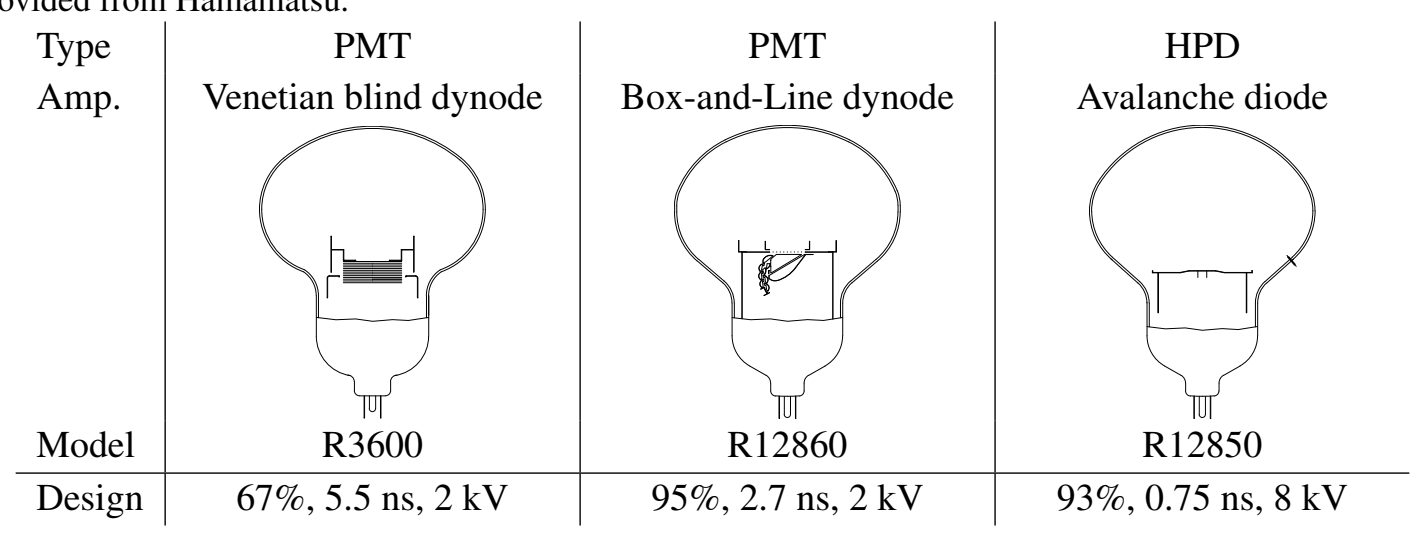

In addition, focusing simulation of electrons indicates an improved collection efficiency (CE). Compared with $73 \%$ CE of the Super-K PMT on average over the $46 \mathrm{~cm}$ area, the Box\&Line PMT reaches $95 \%$ in the same area and HPD gives $93 \%$.

The total detection efficiency of the high-QE Box\&Line PMT is twice as high as the conventional R3600 (Super-K PMT). Figure D shows relative hit counting of single PEs over the whole PMT surface along two directions defined on the dynode. In spite of the asymmetric dynode structure, the response of the Box\&Line PMT is quite uniform.

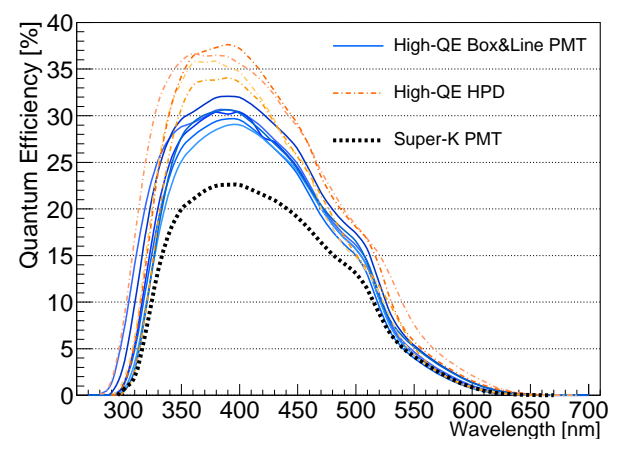

Figure 1: Measured QE spectra of six high-QE Box\&Line PMTs (solid lines), five high-QE HPDs and a typical Super-K PMT (dashed line).

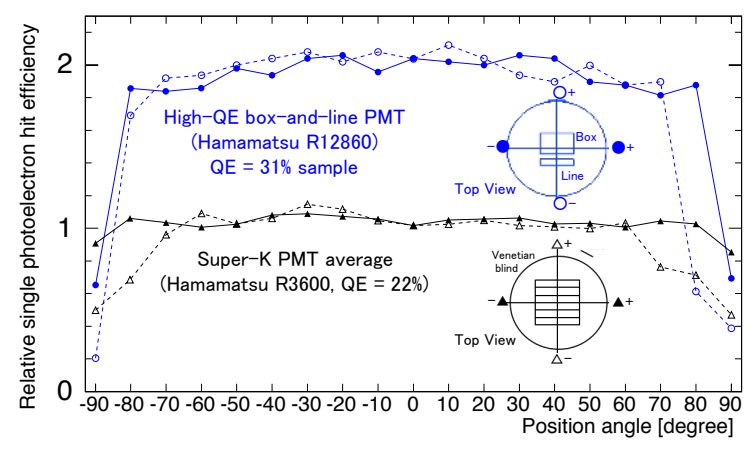

Figure 2: Relative single photoelectron detection efficiency of a Box\&Line PMT to Super-K PMT as a function of the position on the photocathode, where a position angle is defined to be zero at the PMT center and $\pm 90^{\circ}$ at the edges.

Using a diffuser ball as a light source that was placed one meter away from the photo-detector surface, relative counting of single PEs in the overall detection area was measured among PMTs and HPD. The Box\&Line PMT gave a factor of 1.91 compared with Super-K PMT, while 1.76 in the case of HPD. The hit threshold was set at $1 \mathrm{mV}$ in PMTs corresponding to $0.15-0.25 \mathrm{PE}$, while a higher threshold level at $0.5 \mathrm{PE}$ to avoid a noise of preamplifier brought the lower factor of the HPD. 


\subsection{Performance at Single Photoelectron}

Figure [1] shows the charge distribution at single PE for the Super-K PMT (black), Box\&Line PMT (blue) and HPD (red), where the resolution in standard deviation was evaluated to be 53\%, $35 \%$ and $15 \%$, respectively. The HPD resolution is limited by the preamplifier noise because of a large junction capacitance of the $\mathrm{AD}(400 \mathrm{nF})$. As a test, the $\mathrm{AD}$ with segmented channels into two was developed to improve the resolutions with reducing each AD area. Individual HPD readout of the 2-channel HPD showed better resolution of $10 \%$ as shown by a dotted line.

The transit time spread at single PE is given in Figure $\$$ with measured timing at the fixed threshold. It was obtained to be 7.3, 4.1 and $3.6 \mathrm{nsec}$ in FWHM for the Super-K PMT, Box\&Line PMT (blue) and HPD (red), respectively. If the time walk correction is applied using charge, the HPD resolution reached $3.2 \mathrm{nsec}$ in FWHM as shown by the dotted line (pink).

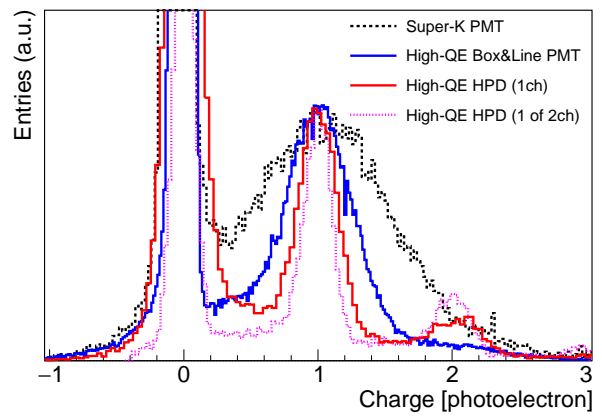

Figure 3: Charge distribution around single PE.

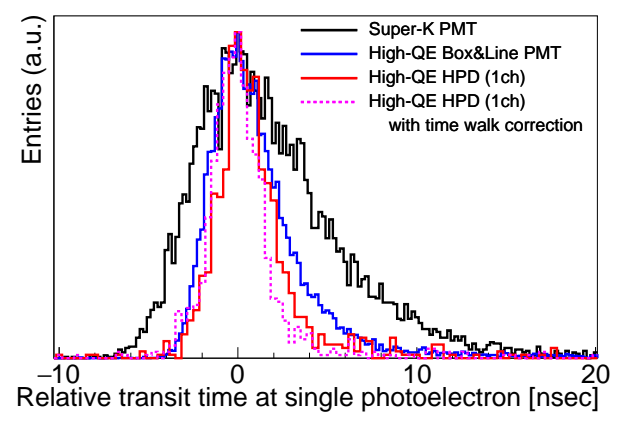

Figure 4: Transit time spread at single PE.

\subsection{Dark Count and After Pulse Rate}

The current typical dark count rate of the Box\&Line PMT is $8 \mathrm{kHz}$, while further reduction is still being tried. It is higher than $4 \mathrm{kHz}$ of the Super-K PMT due to the high QE and CE. The rate of the HPD is similar to that of the Box\&Line PMTs.

The Box\&Line dynode brings a large after pulse rate in general because of its opened structure to the photocathode, but it was lowered by the R\&D. As for the Box\&Line PMT, the number of expected after pulses in a range of $1-45 \mu \mathrm{sec}$ is typically 0.05 for one primary pulse of the single PE intensity. It is comparable with the Super-K PMT that is less than 0.025. The HPD showed a low after pulse rate less than 0.023 .

\section{Validation for a Long and Safe Operation}

\subsection{Proof Test in a Water Cherenkov Detector}

The Hyper-K detector is assumed to run over ten years, thus a long lifetime of the photodetectors is required in Hyper-K. A 200 ton water Cherenkov detector at the Kamioka mine, Japan, was utilized for a long term performance test of new photo-detectors. The tank was constructed for a study of anti-neutrino tagging with gadolinium doped in water, called EGADS [6], and can 
be equipped with 240 photo-detectors in total. For over two years, the proof test using new photodetectors has continued with three Box\&Line PMTs and eight $20 \mathrm{~cm}$ HPDs which has a similar design to the $50 \mathrm{~cm}$ HPDs, in addition to 227 Super-K PMTs.

During recent 1.5 years, the stability of the relative Box\&Line PMT gain to the average of Super-K PMTs was confirmed to be within 2\%. One of the Box\&Line PMTs showed a rate getting higher gradually to around $50 \mathrm{kHz}$. It is probably due to a failure of initial production stage because the condition of the production was surveyed with changing procedures one by one. Other two PMTs showed a stable dark rate in 5\% RMS during the recent 1.5 years.

A prototype of the $50 \mathrm{~cm}$ waterproof HPD was prepared for pretesting before the coming proof test in the 200 ton water tank. It was successfully operated for 20 days in a dark box filled with water.

\subsection{Mechanical Strength}

In 2001 after 5-year operation in Super-K, 6,779 Super-K PMTs (61\% of all) imploded during a maintenance work. It was triggered by an accidental implosion which was transmitted to other PMTs as a pressure pulse. Therefore, a photo-detector cover is necessary to prevent the shock wave pulse from going outside of the cover to stop such a chain reaction absolutely.

In addition, improvement of the bulb design is required to get a high pressure tolerance for use in the $60 \mathrm{~m}$ water depth of Hyper-K that is deeper than $40 \mathrm{~m}$ of Super-K.

\subsubsection{Bulb Strength}

Both Box\&Line PMT and HPD achieved a high pressure tolerance by a safety factor of two for the $60 \mathrm{~m}$ water depth in Hyper-K. The curvature of the bulb was optimized according to a stress analysis, where the weakest part around the neck of the Super-K PMT bulb was changed to get a smooth curvature. Because a broken level is known to be correlated with the glass thickness during the R\&D, the glass thickness was scanned at various points in all of the bulb for a good quality control and selection. Then a validation test was performed on Box\&Line PMTs pressurized up to $1.25 \mathrm{MPa}$ water load, and there was no damage at all of 50 samples. As well, three HPD bulbs were tested and no damage was confirmed at the same level.

\subsubsection{Cover and Validation}

An initial design of the photo-detector cover (Figure \$), made of a $15 \mathrm{~mm}$ UV transparent acrylic window and a $3 \mathrm{~mm}$ stainless steel cover, was established after several tests.

In the usual operation of Hyper-K, the bulb in the cover with several small holes is pressurized by high pressure water. The cover only withstands at the moment when the implosion occurs inside, and should suppress the pressure pulse to outside by a slow water flow through the holes of the cover without breaking.

In a hydrostatic pressure test, the cover packed in a plastic bag was pressurized up to $1 \mathrm{MPa}$ and we confirmed no damage in two samples. Even in higher pressure around 1.2 - 1.5 MPa, the cover kept the shape roughly without crashing, though a buckling analysis showed dent parts appearing consistently.

Then we performed an implosion test in both $60 \mathrm{~m}$ and $80 \mathrm{~m}$ deep water using a vertical shaft filled with water. In one test, a single PMT with the cover was brought to implosion, and in other 
two tests the central PMT with the cover surrounded by eight bare PMTs $(3 \times 3$ alignment in a plane) was imploded. In three sets of test for each $60 \mathrm{~m}$ and $80 \mathrm{~m}$ water level, the cover and all surrounding PMTs survived without damages. The monitoring system consisted of four pressure tourmaline gauges in water, five strain gauges on the cover from top acrylic to cable hole on the stainless steel cover, and a high-speed camera. By the pressure gauge monitor in front of the PMT face, the shock wave pulse was confirmed to be significantly reduced by less than $1 / 100$ suppression by the cover as shown in Figure 6 .

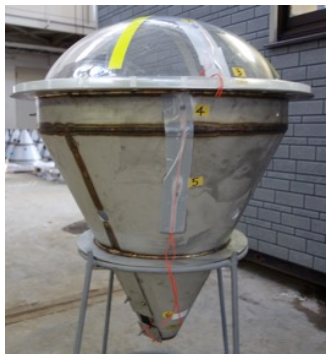

Figure 5: Stainless steel cover and acrylic window attached with five strain gauges.
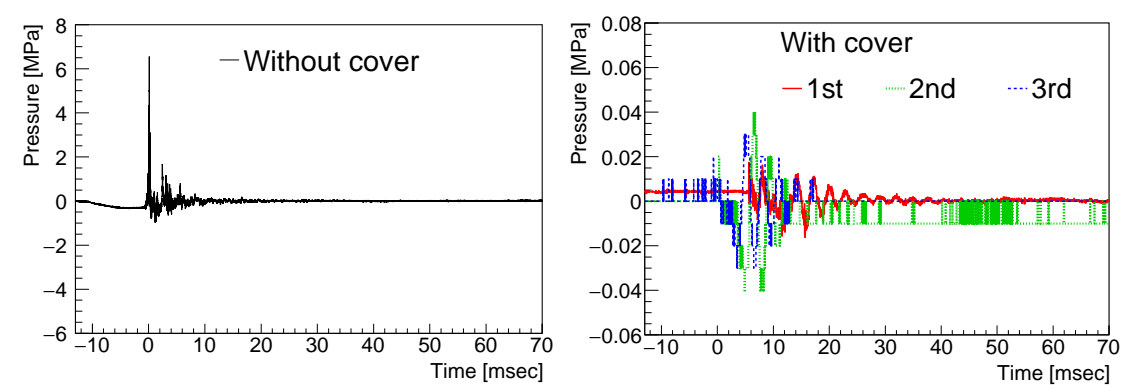

Figure 6: Pressure monitor at $70 \mathrm{~cm}$ ahead of the PMT center in the test without the cover (left) and three tests with the cover (right).

\section{Conclusion}

The high-QE $50 \mathrm{~cm}$ Box\&Line PMT was successfully developed with twice detection efficiency and improved single PE resolutions. Though not all of the evaluations are described here, various performances were confirmed to be sufficient for Hyper-K, such as a wide output range up to 340 PEs within 5\%, or high input frequency up to $87 \mathrm{MHz}$ in the single PE [U]. It was practically established for the long operation over a year in a water Cherenkov detector, and for the high pressure water up to $125 \mathrm{~m}$ water depth. As for the safety measure, the PMT cover was designed and validated with the implosion test even in the $80 \mathrm{~m}$ water depth.

Further high resolutions can be achieved by the $50 \mathrm{~cm}$ HPD. The mechanical characteristics and detection efficiency are similar to the Box\&Line PMTs. The validation of the $50 \mathrm{~cm}$ HPD in the water Cherenkov detector is planned, in addition to the $20 \mathrm{~cm}$ HPDs tested so far.

Hyper-K would bring rich physics results on neutrinos and nucleon decay using the new $50 \mathrm{~cm}$ photo-detector by the twice detection efficiency and twice better resolutions compared with Super$\mathrm{K}$. The operation is planned to start from 2026 year in the earliest case.

\section{References}

[1] Hyper-Kamiokande proto-collaboration: KEK Preprint 2016-21, ICRR-Report-701-2016-1 (2016).

[2] The Super-Kamiokande Collaboration: Nucl. Instrum. Meth. A501 (2003) 418.

[3] H. Kume et al.: Nucl. Instrum. Meth. 205 (1983) 443.

[4] A. Suzuki et al.: Nucl. Instrum. Meth. A329 (1993) 299.

[5] T. Mori for the Super-Kamiokande Collaboration: Nucl. Instrum. Meth. A732 (2013) 316. 